\title{
THE NOSE AS A TARGET OF AIR POLLUTION, PHYSIOLOGICAL ASPECTS AND CLINICAL RELEVANCE OF NASAL TRPA1 (TRANSIENT RECEPTOR POTENTIAL A1) RECEPTORS
}

\author{
Biringerova Z. ${ }^{1}$, Buday T. ${ }^{2}$, Calkovsky V. ${ }^{3}$, Saniova B. ${ }^{1}$, Plevkova J. ${ }^{2}$ \\ ${ }^{1}$ Anesthesiology and Intensive Medicine Clinic of Jessenius Faculty of Medicine, Comenius University and \\ University Hospital in Martin, Slovakia \\ ${ }^{2}$ Department of Pathological Physiology, Jessenius Faculty of Medicine, Comenius University in Martin, Slovakia \\ ${ }^{3}$ Department of Otorhinolaryngology of Jessenius Faculty of Medicine, Comenius University and University \\ Hospital in Martin, Slovakia
}

\begin{abstract}
Worldwide more than several hundred million humans are exposed to severe air pollution, and a significant part of them also smoke. The effect of air pollution on human health has been recognized for many years.

The nose, as the first portal of entry to the respiratory system is constantly exposed to a large volumes of air, which is composed of a mixture of gases, particulate matter and infectious agent, and any material other than physiological amount of oxygen, nitrogen, carbon dioxide and water is considered as pollutant. Being exposed to critical amount of air pollutants, nasal cavity shares sophisticated system of protective and defensive mechanisms regarding the lower airways and lungs, however the highest risk of direct exposure to relevant air pollutants is just within the nose. Air pollution is considered to be responsible for some of the pathological processes affecting airways prone to allergic reactions in predisposed subjects, onset of nasal sensorineural hyperresponsiveness, nonspecific inflammation, sinonasal cancer and definitely, the risk is not limited just to the nasal mucosa, but can proceed toward the lower airways.

The broad spectrum of irritants probably shares very similar molecular background of action. They are capable to activate the cation channenl tranisient receptor potential A1-TRPAl by covalent modification of the channel protein, because many of them are highly reactive and nonstable molecules with possibilities for various chemical reactions. Activation of this channel is responsible for nociceptive reactions mediated by stimulation of afferent trigeminal nerves, retrograde release of tachykinins, activation of parasympathetic afferent drive leading to increase of mucus output and decrease of nasal patency, with subsequent alteration of nasal functions. Based on the recently described neuro-immune bidirectional relationships, air pollutants may be responsible for recruitment of immune cells with infiltration of the nasal mucosa possibly leading to the inflammatory processes and allergies.

Our paper is discussing the nose as the target for air pollution, and focuses on the relevance of TRPAl channel on trigeminal afferents in pollution mediated responses.
\end{abstract}

Key words: nose, air pollution, TRPA1, airways

\section{INTRODUCTION}

Worldwide, more than several hundred million humans are exposed to severe air pollution, and significant amount of them also smoke. The effect of air pollution on human health has been recognized for many years. Dramatic episodes of severe pollution occurred in the Meuse Valley in Belgium in 1930, Donora (Pennsylvania, USA) in 1948 and London, UK in 1952. Majority of pollution of that period was caused by sulphur dioxide derived from coal used for domestic heating. The introduction of the Clean Air Act in the UK in 1956 reduced air pollution as it was followed by the change of domestic heating method. Later coal associated pollution has been replaced by other types of pollutant resulting from the increasing use of liquid petroleum and gas in transport, domestic and industrial settings [1].

Growing interest in indoor pollution has appeared in the early 1980s and 1990s when more than 300 volatile organic compounds were identified as indoor pollutants. They orig-

Address for correspondence:

Assoc. Prof. Jana Plevkova MD, PhD., Department of Pathophysiology, Jessenius Faculty of Medicine in Martin, CU, Sklabinska Str. 26, 03601 Martin, Slovakia

E-mail: plevkova@jfmed.uniba.sk, 
inate from building materials, combustion fumes, cleaning compounds, paints and stains in addition to tobacco smoke [2]. This coincided with the description of sick building syndrome, characterized by eye, nose and throat irritation, headache, mental fatigue, and respiratory distress affecting some sensitive individuals after the exposure to indoor environment.

Inhalation of pollutants is associated with adverse cardiovascular and respiratory reactions and may lead to an increase in mortality. The nose, as the first portal of entry to the respiratory system is constantly exposed to a large volumes of air, which is composed of a mixture of gases, particulate matter and infectious agents, and any material other than physiological amount of oxygen, nitrogen, carbon dioxide and water is considered as pollutant [3]. Two critical issues in understanding the etiology of air pollution caused airway damage are the identification of the responsible chemicals and the identification of their receptors on epithelial cells and sensory nerves that innervate these areas.

Much progress has been made as recent studies point to a role for TRPAl channels in response to pollutants, whether they originate from cigarette smoke, oxidizing agents, or from other irritants in the air. Our rewiev is focused on the importance of the nose, as the target for air pollution and the relevance of TRPA1 channel in responses of the airways to the common airborn pollutants [4].

\section{NOSE AND IT S FUNCTIONS}

Nasal cavity is surrounded by bone and divided into halves by the septum. The two separate airways extent from the anterior nares to the posterior ones, where they are united to a single airway at the nasopharyngeal level. The nose has an external visible part surrounding the vestibule and an internal part containing the main nasal cavity. The nasal vestibules are trumpet-shaped orifices, narrowing towards the main nasal cavity. In the main nasal cavity three bony shelves project from lateral wall on each side, the nasal turbinates. The turbinates increase the surface of the nasal mucosa, while at the same time, they narrow the lumen. This design facilitates the nasal functions [5].

The nasal passages are the first parts of the conducting airways carrying the air from the atmosphere toward the lungs, contributing significantly to the humidification and heating of the air. The shape of the nasal cavity which bends and has differences in lumen area, causes turbulent flow of the air and promotes deposition and trapping of particles onto the nasal mucosa. Therefore the air that enters the lower airways is filtered and conditioned. Nasal mucosa is also the site of immune reactions, nasal sinuses are the site of NO production, and the system of cavities within the skull shares the spaces allowing resonance, thus giving characteristic modulation to the voice [6].

In this paper, we would like to focus on those functions which may significantly reduce the hazards for lower airways caused by inadequate conditions of inspired air - mainly the presence of airborn irritants, which contributes to high respiratory morbidity and for sure, can promote exacerbation of chronic airway diseases as chronic obstructive airway disease (COPD), allergic rhinitis or bronchial asthma. It has been shown that impairment of nasal functions significantly influence the functions of lower airways both in humans [7] and in animal models [8].

\section{PROTECTON AGAINST AIR POLLUTION IN UPPER AIRWAYS}

Logically, if the nose shares protective function regarding the lower airways it must be equipped with some possibilities for such a defense. These mechanisms can have defensive or protective role, and could be of reflex and non reflex type.

The general idea of the protective mechanisms is to prevent penetration of dangerous noxae to the respiratory system (for example apnoeic reflex, which does not allow inhalation of the hazards as the breathing is suppressed at the end of expiration phase). Defensive 
mechanisms are activated if any relevant noxa has penetrated to the respiratory system or has been produced (mucus) within the respiratory system, and there is a need to expel it out from the respiratory regions (cough, sneezing) [9].

First important nasal function regarding the airway defense is filtration. Material accompanying the airstream tends to impact on the mucus surface. It is a function of the aerodynamic equivalent diameter (AED). Approximatelly $80 \%$ of material with an AED of $>9 \mu \mathrm{m}$, $50 \%$ with an AED of $2-9 \mu \mathrm{m}$ and $40 \%$ with an AED of $<2 \mu \mathrm{m}$ stick to the nasal wall. The rest finds its way into the lower respiratory tract. In addition, some particles are hygroscopic and tend to accumulate water along the way. This enhances their chances of sticking to the nasal wall. Although this process is effective in protection of the lower airways from relevant amount particulate mater with an AED $>9 \mu \mathrm{m}$, it exposes the nasal mucosa to constant stream of irritants. Nasal exposure to such pollution may promote onset and development of diseases in individuals constatntly exposed to polluted air [10].

Filtration of inspired air is promoted by the specific pattern of air blowing through the nose, which is turbulent. This turbulent flow through nasal passages allows the closest contact of the air with mucosal surface promoting deposition of particles onto the mucus. There are important mechanisms which also enhace that deposition, especially electrostatic interactions between the charge of particles and mucosal surface and impingement.

Impingement is the process allowing deposition of particles onto the nasal wall which is caused by collision between the airstream and the sites of the bending or narrowing of the nasal passages and also at the sites, where the airstream gets different direction [6]. There are two of such places within the upper airways - the nasal vestibule and posterior wall of nasopharyx.

Particles, which were trapped onto the mucosal surface, are effectivelly eliminated from the nasal cavity by the mucociliary transport (MCT) fig.1. A thin blanket of mucus covers the nasal epithelium. It consists of two layers: a low viscosity layer enveloping the cilia and a more viscous layer riding on the sol phase. Nasal mucus is derived from goblet cells, seron-mucus and serous glands, exudate from blood vessels and tears. The mucus contains a lot of molecules with immunomodulatory and antibacterial action including lysozyme, lactoferin, cytokines and immunoglobulines. Particles and substances trapped or disolved in the mucus are swallowed and are then inactivated and destroyed by enzymes in the gastrointestinal tract [11]. The cilia beat with varying rate and propelling speed is usually 6 $\mathrm{mm} / \mathrm{min}$. The function of MCT is very important in imunity for two reasons: it acts as a mechanical escalator removing noxious substances and particles and it actively participates in immune reactions through the mediator it contains.

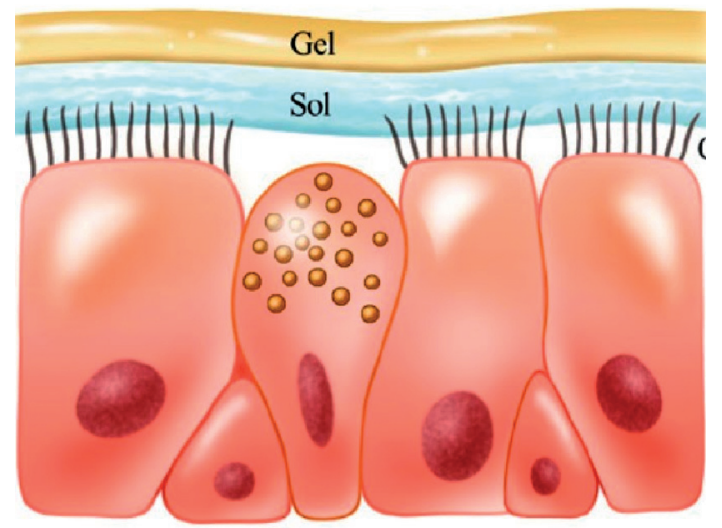

Fig. 1 The mucus layer on the surface of nasal mucosa, and cilia of respiratory epithelium, which belong to the protective and defensive airway mechanisms as a components of mucociliary transport. 
MCT in the nose is relatively resistant to climatic extremes, commonly present environmental pollutants, wide variations of $\mathrm{pH}$, excess of corpuscular pollutants and most of intranasal local treatments. Decreased activity of nasal MCT could be induced by benzalconium chloride (a component of certain drugs), detergent sprays, hypotonic solutions, as a consequence of exsication of the nasal mucosal surface and finally due to viral infections. Cosequence of its reduced effectiveness is increased risk for the mucosa to be infected by any viral primoinfection, or secondary bacterial invasion after viral primoinfection. Recovery of mucociliary funtions after rhinits is completed in couple of weeks [6].

\section{WHAT HAPPENS IF THE NOSE IS EXPOSED TO AIRBORN IRRITANTS?}

We have mentioned mechanisms of non-reflex origin which paricipate in the protection of the respiratory system, but what actually happend if the nasal mucosa is exposed to the airborn irritants? Trigeminal excitation by airborn pollutants initiates the sensation of irritation, pain, lacrimation and sneezing [12]. Sensory-autonomic parasympathetic efferent reflex pathways induce secretions from nasal, lacrimatory, and salivary glands, and the dilation of vessels in the nasal mucosa and sinuses. Neuropeptides, such as substance P and CGRP (calcitonin gene related peptide), released from chemically stimulated nerve endings, promote neurogenic inflammatory vasodilation and leakage, contributing to narrowing or obstruction of the nasal passages [13]. Exposure to airborn pollutants may elicit protective and defensive reflexes such as apnoea, sniffing and sneezing. The role of apnoeic reflex is to prevent inhalation of potential hazzards deeper to the respiratory system. It could be elicited as a response to the cold (diving response), strong chemical irritants like xylol, ammonia, chlorine or cigarette smoke and also some kind of mechanical stimulation of the nasal mucosa [9]. The breathing stops at the end of expiration and new breathing cycle is abolished. Sniffing is considered to be a semireflex process. It is characterized by several brief strong inspirations, which are redirecting the air stream to the neibourghood of the olfactory region. It also promotes cleaning of the nose, as the mucus and particles trapped onto its surface are removed by these strong inspiratory efforts to the nasopharynx and pharynx, from where they could be expelled, or swallowed. Sneezing is defensive reflex, which also could be elicited by stimulation of trigeminal afferents by airborn pollutants. It is also common symptom of rhinitis, because nasal nerves are stimulated by inflammatory mediators and by the excess of mucus, as well [14, 15]. Afferent information is carried out via trigeminal nerve to the sensitive trigeminal nucleus. If the stimulus is strong enough to elicite sneezing, then the sneezing pattern and motor output are generated by central neuronal network. It starts with preparatory inspiration through the mouth - to prevent the aspiration of the hazzard, which has initiated it to more distal airways. Later the glottis is closed with simultaneous activation of expiratory muscles, which allows formation of high intrathoracic pressure. Finally the glottis is open, and the compressed air blows out through the nose to expel the mucus and noxae which have initiated this reflex.

Other consequences of the exposure to irritants are local reflex reactions which are called axon reflexes, and naso-nasal reflexes. Stimulation of afferent nerve endings (mainly $\mathrm{C}$ fibres) leads to retrograde release of neuromediators - tachykinins - substance P, CGRP, VIP (vasoactive intestinal peptide), NPY (neuropeptide Y), which have a diversity of effects on the nasal mucosa, generally known as a neurogenic inflammation [16]. They are capable to induce vasodilatation, increase glandular output and increase vascular permeability with plasma leackage. General idea of this reaction is to dilute the irritant in the superficial mucosal fluids and enhance its removal. The danger of axonal reflex is that continual exposure to irritants could significanlty contribute to prolonged inflammatory process within the upper airways based on neurogenic inflammation.

Nasonasal reflexes participate mainly in the regulation of the vascular tone, congestion of mucosal sinuses and mucus output by glands. The afferent arm is secured by the trigeminal afferents, and the efferent drive is provided by the autonomic fibres. In the response to 
irritants, mainly the parasympathetic nerves are called upon the action, and their main effects are similar to those provided by tachykinis - so the exposure to irritants induces parasympathetic nerves mediated hypersecretion and vasodilatation with venous sinus engorgement [12]. Stimuation of the trigeminal afferents by relevant stimuli (cold, chemical irritants, mechanical probing) may induce also strong cardiovascular responses. For example, apnoea is followed by bradycardia, hypertension with redistribution of circulating blood [17]. Stimulation of nasal afferent is believd to influence resistance of lower airways. This mechanism is called nasobronchial reflex. Some scientists confirmed that acute nasal provocation challenges are followed by decrease of lower airway patency [18] with limited expiration flows. If the nose is constantly exposed to irritants, these reflexes may significantly contribute to the airflow limitation thus leading to symptoms due to bronchoconstriction such is dyspnoe or wheezing.

\section{CONSEQUENCES INDUCED BY NASAL EXPOSURE TO AIRBORN IRRITANTS}

If the subjects are exposed to air pollution and they are breathing through the nose, what is preffered pattern of inspiration for subjects in rest conditions, then the nose is the first site of the respiratory system, which is affected by the pollutants themselves.

As we mentioned before, immediate responses to the pollutant exposure involve sensory nerve activation and its consequences such are the reflex responses, retrograde tachykinins release and finaly activation of parasympathetic afferent drive. These machanisms are responsible for typical symptoms which follow exposure to irritants, like irritation, sneezing, nasal discharge, lacrimation and nasal obstruction. These smptoms are present in case the action of pollutant is being limited to the nasal mucosa. However effective is the nasal cavity regarding the protective and defensive mechanisms, some of the pollutants can definitelly reach the lower airways.

The question is, what is the impact of well defined airborn pollutants on the nose? This effect has been identified for most common pollutants. For example ozone $-\mathrm{O}_{3}$ which is formed in the atmosphere through complex photochemical reaction sequence that requires reactive hydrocarbons, nitrogen dioxide and sunlight [19]. It has been shown that exposition to ozone causes inflammatory response in the nasal mucosa indicated by an influx of neutrophils, and the inflammatory markers as eosinophilic cationic protein, albumin and leukocytes in the nasal lavage fluids correlate with increase of ambient ozone levels.

Nitrogen oxides have also impact on the nasal mucosa. In the atmosphere, NO is gradually converted to $\mathrm{NO}_{2}$ and, like ozone, $\mathrm{NO}_{\mathrm{x}}$ are a product of combustion of petroleum. The effect of $\mathrm{NO}_{2}$ was determined in subjects exposed to air and $400 \mathrm{ppb} \mathrm{NO}_{2}$ for 6 hours and it was found that exposure to $\mathrm{NO}_{2}$ did not affect the count of immune cells within the mucosa, but when the $\mathrm{NO}_{2}$ or air exposure was followed by the allergen challenge, it was documented that allergen induced immune cells recruitment was much stronger in $\mathrm{NO}_{2}$ rather than air treated group [20].

$\mathrm{SO}_{2}$ is a by-product of coal burning for domestic and industrial heating. The effect of $\mathrm{SO}_{2}$ on nasal mucosa was determined after exposure to $750 \mathrm{ppb}$. Nasal mucosal biopsies were analyzed and the authors documented that $\mathrm{SO}_{2}$ exposure caused a cilia compounding with changes of the ultra structure of ciliary membrane.

The acute effects of outdoor or indoor exposure to various fumes and particulate matter have been demonstrated. It has been reported, that deposition of pollutant particles onto the nasal mucoa leads to the recruitment of immune cells, and may induce T-helper cell like inflammation [21]. Growing evidence also indicates that tobacoo smoke has significant effect on the nose and sinuses. It has been shown, that cigarette smoking or exposure of children to parental smoking may lead to increase risk for allergic rhinitis, nonspecific nasal mucosal inflammation and even sinonasal cancer. Data from epidemiological studies have shown that allergy has increased since the 1960. This increase has been linked to the effects of pollution on the airways by many authors, however some of the literature rewievs spec- 
ulate about clear direct evidence, which could prove the relationship between air pollution and allergy [22]. Some of the recent papers regarding occupational allergies in subjects who had been exposed to air pollutants at workplace and studies comparing the respiratory function of citizens living in industrial/traffic areas vs those who live in country-side, or less polluted areas clearly shows that exposure to air pollution enhace respiratory morbidity [23].

\section{IS THERE ANY RELEVANT SENSOR TO DETECT AIR POLLUTION?}

The airways are innervated by both chemosensory nerves detecting a large variety of noxious chemicals and mechanosensory nerves that constantly measure the tension of airway tissue and participate in respiratory feedback control. Trigeminal chemosensory nerve endings in the nasal mucosa are in the first line of defense against noxious challenges.

Among many classes of nerve fibers are the polymodal nociceptors (PMNs). These unmyelinated neurons send signals that cause the perception of irritation and/or pain in response to potentially damaging thermal, mechanical, and chemical stimuli. Their activation induces protective reflexes and nocifensive behaviors. Although there are many types of PMNs, the most common are those that are activated by capsaicin through its receptor, transient receptor potential cation channel, family V, member 1 (TRPV1). TRPV1 is a member of the TRP family that are all inhibited by the polyvalent cationic dye and ion channel blocker ruthenium red, but specific antagonists exist for individual TRP channels. When capsaicin-sensitive neurons are activated, they transmit nociceptive information to upstream relay centers within the CNS that are associated with pain perception and these neurons also release proinflammatory mediators via retrograde signaling [24]. The major portion of nociceptive trigeminal fibers express TRPV1 channel and they are called capsaicin sensitive fibers. It had been shown that reaction to cigarete smoke in experiment, which was believed to be mediated via TRPV1 channel, was not completely inhibited by selective antagonist capsazepine. This was a new result, which focused on TRPV1-independent mechanism in the phenomenon of cigarette smoke induced airway irritation and that event had pointed the critical role of another TRP channel in airway chemosensation.

\section{A NEW MEMBER OF TRP FAMILY}

An initial breakthrough was made when pharmacological experiments revealed that acrolein, the noxious unsaturated aldehyde enriched in photochemical smog and smoke is a potent agonist of human and murine TRPAl channels. Cultured sensory neurons from TRPA1-deficient mice lacked any response to this irritant, suggesting that TRPA1 is the sole chemosensory receptor for acrolein [25], fig. 2.

This transient receptor potential channel belongs to the TRP group, family A, member 1 (TRPA1). TRPA1, like TRPV1, is expressed by trigeminal neurons and, moreover, both channels are most often found on the same neuron. It means that activation of TRPA1 will likely exert effects similar to those observed following the activation of TRPV1. The channels are generally activated by different agonists (an exception is allicin, the pungent ingredient in garlic, which activates both TRPA1 and TRPV1). TRPA1 is exclusively activated by mustard oil, cinnamaldehyde, lipids, chlorine, the pollutant acrolein, and some endogenous molecules. Calcium ions are essential co-activators of TRPAl as removal of $\mathrm{Ca}^{2+}$ dramatically reduced the potency of mustard oil to activate TRPA1 and slowed channel activation kinetics. TRPAl is not inhibited by capsazepine but rather by the specific antagonists HC-030031 and AP 18 [26]. In sensory neurons, TRPAl was first identified as a cold-sensitive ion channel in a small subset of sensory neurons. However, later studies showed that TRPA1 expression is more widespread, encompassing $20-35 \%$ of sensory neurons. Pharmacological experiments revealed that TRPAl is the sensory neuronal receptor for mustard oil (allyl isothiocyanate), the pungent ingredient in mustard and wasabi. Similar to capsaicin, mus- 


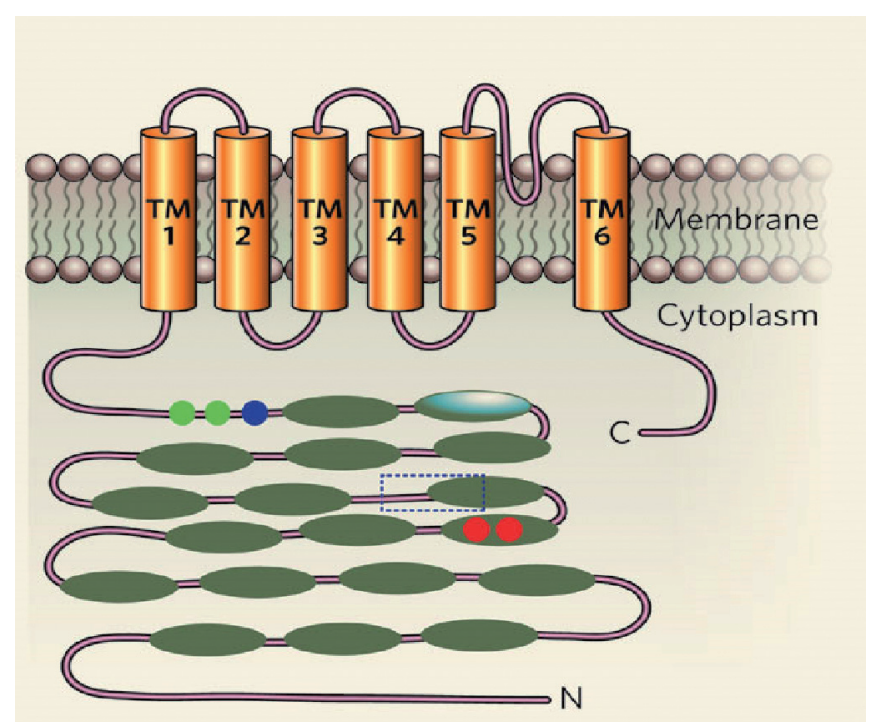

Fig. 2 Structure of TRPAl cation channel, which is believed to be the target for mort important air pollutants entering airways.

tard oil activates peripheral C-fibers, causing acute pain, thermal and mechanical hyperalgesia, and neurogenic inflammation. Recent studies identified additional pungent natural products as TRPA1 agonists. These include cinnamon aldehyde from cinnamon, allicin and diallyl sulfides from garlic and onions, carvacrol in oregano, isovalleral, a fungal deterrent [27]. The properties of TRPA1 show parallels with those of the proposed reactive airway irritant receptor. Functional imaging and electrophysiological studies showed that TRPAl is expressed in a subpopulation of TRPV1-expressing C-fiber neurons and is permeable to calcium. This finding is in agreement with previous studies showing that reactive airway irritants activate influx of calcium in a subset of capsaicin sensitive neurons. Then, TRPA1 is blocked by ruthenium red known to antagonize irritant-induced reactions. Capsaicin pretreatment makes C-fibers insensitive to both mustard oil and chemical airway irritants and finally AITC (allylisothiocyanate) - mustard oil itself is a potent upper airway irritant. Taken together, these facts encouraged closer examination of the role of TRPAl in airway chemical sensing [28].

TRPA1 is not only sensitive to electrophiles, but is also activated by oxidizing agents entering the airways including the hypochlorite $(\mathrm{OCl}-)$, the oxidative mediator of airway irritant - chlorine, which is a common chemical, used in industrial processes and for disinfection. Hydrogen peroxide, another oxidizing agent known to excite airway nerve fibers and to induce respiratory depression also stimulates TRPA1. Results of the studies have been confirmed on TRPAl lack mice and using selective TRPAl antagonists. In summary, these recent studies have shown that TRPAl is essential for chemical sensing of oxidants and electrophilic agents in the airways, initiating physiological responses such as respiratory depression and neurogenic inflammation [25].

As it was mentioned before, TRPA1 is activated by a large variety of reactive irritants, including acrolein, a major constituent of tobacco smoke, allicin, mustard oil, and cinnamaldehyde, tear gas, the reactive oxygen species, hydrogen peroxide and hydroxyl radical, hypochlorite, chlorine, formaldehyde, isovelleral, sesquiterpene and other molecules. How can a single receptor cover such broad chemical space?

Recent structure function studies provided a potential explanation for the diversity of TRPA1 agonists. These studies suggest that TRPA1 is activated through covalent modifica- 
tion of the channel protein. Most TRPAl agonists are chemically unstable in biological environments and can undergo chemical reactions with proteins, lipids, nucleic acids, and metabolic products [29]. Covalent modification implies that dose-response relationships and activation kinetics of TRPAl do not conform to standard pharmacological paradigms and are highly dependent on the chemical status of the cellular and tissue environment. For any given chemical, TRPAl agonist activity will depend on the reversible or irreversible nature of the chemical bonds formed and on agonist membrane permeability. With each breath, more reactive agonist is delivered, leading to an increase in covalent modifications and heightened TRPAl activity. This cumulative effect may result in robust TRPAl-induced irritation even at low subacute exposure levels, for example during periods of increased photochemical smog exposures, or low level indoor air pollution. Once irreversibly modified, channels may remain active for extended periods of time even when the irritant stimulus is removed [30]. Oxidative stress is a hallmark of most acute and chronic inflammatory airway diseases, but ROS (rective oxygen species) are also produced during oxidant exposures and through catalysis by inhaled toxic particles. Exposure of cellular membranes to inflammatory ROS, hypochlorite, or exogenous oxidants causes membrane lipid peroxidation, producing electrophilic reactive mediators such as 4-hydroxy-2-nonenal (HNE), 4-oxo-2-nonenal (ONE), as well as cyclopentenone prostaglandins and isoprostanes. Exogenously applied HNE, ONE, and cyclopentenone prostaglandins activated TRPA1 in heterologous cells, neurons, and nerve fibers and induced TRPA1- dependent nocifensive behavior in mice [29].

\section{CONCLUSIONS}

Twenty years ago, Brooks coined the term „reactive airway dysfunction syndrome“ (RADS), which he defined as symptoms mimicking asthma within 24 hours after single massive chemical exposure [31]. Exposures to high levels of TRPA1 agonists, including chlorine and aldehydes, often induce RADS characterized by asthma-like symptoms such as cough, wheezing, chest tightness, and dyspnoea and heightened sensitivity to chemical and physical stimuli, including the initial sensitizing stimulus [32]. The multiple chemical sensitivity of TRPA1 can readily explain the broad chemical sensitivity observed in RADS patients.

The nose as the very first site of the respiratory system which is exposed to airborn irritants may play critical role in airway protection, however, continual exposure to pollutants may initiate such processes, which in turn can potentiate onset and develepoment of diseases such is allergic rhinitis, nonallergic rhinitis, bronchial hyperreactivity syndroma, bronchial asthma and upper airway cough syndrome [7]. Last mentioned problems are well defined as being, most likely, concequences of upper vs lower airways bidirectional relationships. Some of them are also resistant to common rhinitis and/ or asthma treatment.

If these reactions are mediated by TRPAl channel leading to prolonged hypersensitivity to multiple reactive chemicals, then using TRPAl antagonist may be of high clinical importance, especially if it can significantly reduce, or abolish irritant induced reaction, and irtirant induced pathological process. TRPAl antagonists may be useful for blocking the exaggerated chemosensory responses accompanying these conditions. Post exposure treatment with TRP channel antagonists may reduce sensory irritation and potentially, prevent adverse long-term health effects elicited by neurogenic inflammatory mechanisms.

\section{REFERENCES}

1. Davies RJ; Devalia JL. Air pollution and airway epithelial cells. Agents Actions Supplement 1993; 43: 87-96

2. Koren HS, Devlin RB. Human upper respiratory tract responses to inhaled pollutants with emphasis on nasal lavage. Ann NY Acad Sci 1992; 641: 215-224

3. Leopold DA. Pollution, the nose and sinuses. Otolaryngol Head Neck Surg 1992; 106: 713-719.

4. Simon SA, Liedtke W. How irritating: the role of TRPAl in sensing cigarette smoke and aerogenic oxidants in the airways. Journal of Clin Invest, 2008; 118, 2383-2386 
5. Gaga M, Vignola AM, Chanez P. Upper and lower airways: similarities and differences. European Respiratory Monograph 2001; 6, 1-15.

6. Plevkova J, Tatar M. Physiology and pathophysiology of the nose, nasal hyper responsiveness (in Slovak), Cs fyziologie 2002; 51, 1: 28-36

7. Pecova R, Zucha J, Péč M, Neuschlová M, Hanzel P, Tatár M. Cough rensitivity testing in seasonal allergic rhinitis patients and healthy volunteers. J Physiol Pharmacol 2008, 59:6, 557-564

8. Brozmanova M, Bartos V, Plank L, Tatar M. Experimental allergic rhinitis related cough and airway eosinophilia in sensitized guinea pigs. J Physiol Pharmacol 2007; 58:5, 57-65.

9. Korpas J, Tomori Z. Cough and other respiratory reflexes. Veda, vydavatelstvo SAV, 1979, $368 \mathrm{~s}$.

10. Samir M, Magdy S, el Fetoh AA. Air pollution in relation to allergic and nonallergic rhinitis. Arch Otolaryngol Head neck Surgery, 1997; 123: 746-748.

11. Kupferberg SB, Bent JP, Porubsky ES. The evaluation of ciliary function: electron versus light microscopy. Amm J Rhinol 1998; 12: 199-201.

12. Sarin S, Undem B, Sanico A, Togias A. The role of the nervous system in rhinitis. J Allergy Clin Immunol, 2006; 118: 999-1016.

13. Taylor - Clark T. Insight into the mechanisms of histamine-induced inflammation in the nasal mucosa. Pulmonary Pharmacol Ther, 2008; 21: 455-460.

14. Antosova M, Turcan T, Strapkova A, Nosalova G. Inhibition of guanylyl cyclase in the airways heperreactivity. Bratisl lek listy, 2005; 8-9.

15. Brozmanova M, Plevkova J, Bartoa V, Plank L, Tatar M. Antileukotriene treatment and allergic rhinitis - related cough in guinea pigs. J Physiol Pharmacol 2005; 56(4): 21-30

16. Barnes PJ. Neurogenic inflammation in the airways. Respir Physiol., 125, 2001, 145-154.

17. Angel James J \& Daly MB. Reflex respiratory and cardiovascular effects of stimulation of receptors in the nose of the dog. J Physiol., 1972, 220, 637-696.

18. Plevkova J, Kollarik M, Brozmanova M, Revallo M, Varechova S, Tatar M. Modulation of experimentally induced cough by stimulation of nasal mucosa in cats andguinea pigs. Respir Physiol Neurobiol 2004; 142 : 225-235.

19. Lipman M, Schlesinger RB. Toxicological bases for the setting of health- related air pollution standards. Annu Rev Public health 2000; 21:309-333.

20. Wang JH, Devalia JL, Duddle JM, Hamilton SA, Davies RJ. Effect of six hour exposure to nitrogen dioxide on early phase nasal responses to allergen challenge on patients with a history of seasonal allergic rhinitis. $J$ Allergy Clin immunol 1995; 96:669-676.

21. Samet JM, Spiezer FE. Assesment of health effects in epidemiological studies of air pollution. Environ Health Perspect 1993; 4:149-154.

22. Benninger MS. The impact of cigarette smoking and environmental tobacoo smoke on nasal and sinus disease: review of the literature. Am J Rhinol 1999; 13: 435- 437.

23. Varechova S, Mikler J, Murgas D, Dragula D, Banovcin P, Hanacek J. Cough reflex sensitivity in children with suspected and confirmed gastroesophageal reflux disease. J Physiol Pharmacol 2007; 58:5, 717-27.

24. Taylor-Clark TE, Kollarik M, MacGlashan E, Donald W, Undem BJ. Nasal sensory nerves responding to histamine and capsaicin. J Allergy Clin Immunol 2005; 116: 1282- 1288.

25. Bautista Dm, Jordt SE, Nikai T, Tsuruda PR, Read Aj, Poblete J, Yamoah EN, Basbaum Ai, Julius D. TRPA1 mediates the inflammatory action of environmental irritants and proalgesic agents. 2006; Cell, 124:1269-1282

26. Bessac BF, Jordt SE. Breathtaking TRP channels: TRPA1 and TRPV1 in airway chemosensation and reflex control. Physiology 2008; 23, 360-370

27. Gerhold KA \& Bautista DM. TRPA1: irritant receptor of the airways. J Physiol 2008, 586.14, 3303

28. Anderson DA, Gentry C, Moss S, Bevan S. Transient receptor potential Al is a sensory receptor for multiple products in oxidative stress, J Neurosci 2008; 28: 2485-2494.

29. Hinman A, Chuang HH, Bautista DM, Julius D. TRP channel activation by reversible covalent modification. Proc Natl Acad Sci USA 2006; 103:195664-19568

30. Bessac BF, Sivula M, von Hehn CA, Caceres AI, Escalera J, Cohn L, Jordt SE. TRPA1 is a major oxidant sensor in murine airway sensory neurons. J Clin Invest 2008; 118: 1899-1910

31. Moriss JB, Wilkie WS, Shusterman DS. Acute respiratory responses of the mouse to chlorine. Toxicol Sci 2005; 83: 380-387.

32. Brooks SM, Weiss MA, Bernstein IL. Reactive airway dysfunction syndrome (RADS). Persistent asthma syndrome after high -level irritant exposure. Chest 1985, 88: 376-384.

33. Shakeri MS, Dick FD, Ayres JG. Which agents cause reactive airway dysfunction syndrome? A systemic review. Occupational Medicine 2008, 58:205-211.

Received: April, 7, 2011

Accepted: May, 15, 2011 